\title{
Gerontomotricidade e o idoso praticante de atividades físicas: um estudo teórico
}

\author{
Gerontomotricity and the elderly practice of physical activities: \\ a theoretical study
}

Vinícius Bittencourt Miranda'

\section{RESUMO}

Tem-se como proposta apresentar conceituações sobre o envelhecimento, enumerando aspectos decorrentes deste processo natural ao ser humano, tratando de questões neuromusculares, cardiorrespiratórias, da composição corporal, da endocrinologia e de fatores patológicos do envelhecimento inerentes à essas ocorrências, abordando a realização de atividades físicas durante a velhice, apontando as consequências dessas ao indivíduo idoso. A seguir, aborda-se a psicomotricidade e a gerontomotricidade, correlacionando essas informações com a realização de atividades físicas na concepção do ser idoso. As considerações finais unem essas perspectivas sob a ótica do autor, verificando a importância da realização das atividades psicomotoras na melhora da qualidade de vida do idoso, bem como elucidando a responsabilidade do profissional de Educação física que orienta esse programa de treinamento, criando um impacto social positivo na saúde física e mental de seus alunos, fundamental na terceira idade.

\section{PALAVRAS-CHAVE}

Idoso; Atividade Física; Envelhecimento; Gerontomotricidade. 


\section{ABSTRACT}

The purpose of this study is to present concepts about aging, enumerating aspects of this natural process to the human being, dealing with neuromuscular, cardiorespiratory, body composition, endocrinology and pathological aging factors inherent to these occurrences, addressing the accomplishment of activities during the old age, pointing out the consequences of these to the elderly individual. Next, psychomotricity and gerontomotricity are discussed, correlating this information with the accomplishment of physical activities in the conception of the elderly. The final considerations combine these perspectives from the perspective of the author, verifying the importance of the performance of the psychomotor activities in the improvement of the quality of life of the elderly, as well as elucidating the responsibility of the Physical Education professional who guides this training program, creating a positive social impact in physical and mental health of its students, fundamental in the third age.

\section{KEYWORDS}

Aged; Physical Activity; Aging; Gerontomotricity. 


\section{INTRODUÇÃO}

O aumento da longevidade está associado à diminuição das doenças, principalmente em países de alta renda per capita, e à uma prática de hábitos de vida saudáveis. Desta forma, devemos encarar o envelhecimento como uma fase longa e cada vez mais integrante do processo de vida do indivíduo. Durante esse processo, ocorrem no organismo humano alterações fisiológicas, mecânicas e mentais ou psicológicas. Recomendações de especialistas, em busca de atenuar essas ocorrências, incluem a execução de um programa de atividade física, visto que esta é um importante aliado para minimizar os impactos causados pelo envelhecimento, sendo também fator relevante no aspecto da vida social da população idosa. Este artigo teve como proposta analisar e identificar na literatura, principalmente em artigos científicos originais e em livros, os efeitos da atividade física nos aspectos das funções cognitiva e psicomotora dos idosos, bem como orientar a elaboração de um programa de atividades psicomotoras, com ênfase na gerontomotricidade como estratégia na manutenção dessas aptidões.

Os procedimentos metodológicos que foram utilizados nesta revisão narrativa da literatura sobre o tema consistiram em um levantamento sobre a produção científica disponível através de descritores como: envelhecimento; gerontomotricidade; idosos; atividade física; nas bases de dados em seu sistema de busca na Internet citadas por Scopus, Science, Direct, PubMed, Scielo, além da literatura disponível de forma não virtual ou de forma física. Nas palavras de Andrade (2007, p.58), a revisão bibliográfica "ilumina o problema com a discussão de novos enfoques, dados, informações, esclarecendo melhor as questões sob análise e permitindo construir a fundamentação teórica da pesquisa". Por assim ser, esse amparo metodológico serve para catalogar estudos confiáveis, proporcionar sínteses e estabelecer fontes para novos estudos. Por meio desses descritores foram encontrados 48 artigos, dos quais 25 foram utilizados por terem sido publicados após o ano de 2000. Além disso, foram utilizadas 2 dissertações de mestrado, 24 obras literárias e uma fonte digital.

Sendo assim, além da introdução, este artigo se constitui de uma abordagem do processo do enveIhecimento, a relevância da prática de atividades físicas pelos idosos nos aspectos psicomotores e a gerontomotricidade como ciência onde verifica-se a importância do conhecimento e prática das atividades psicomotoras ao público idoso, que podem proporcionar um estado completo de bem-estar físico, social e psicológico à esta camada da população, além de sugestões para próximos estudos.

\section{ENVELHECIMENTO}

O envelhecimento é um fenômeno complexo e variável, que ocorre inevitavelmente com o passar do tempo, cabendo às ciências da saúde desenvolver seu estudo sob uma perspectiva interdisciplinar. Nahas (2006) conceitua o envelhecimento como um acontecimento de forma gradual, universal e irreversível, acarretando perdas funcionais no organismo. Esse processo é acompanhado por várias alterações orgânicas como, por exemplo, a redução do equilíbrio estático e dinâmico e da mobilidade articular, modificações das funções fisiológicas (respiratória e circulatória) e transformações psicológicas (maior vulnerabilidade à ansiedade e à depressão).

O marcador cronológico talvez não seja o mais preciso para mensurar os aspectos do envelhecimento. Para uma melhor compreensão e tomada de novas atitudes sobre o envelhecer, devemos analisar os aspectos intercorrentes e, a partir disso, propor alternativas que se complementem e atenuem este processo. As divisões cronológicas da vida humana não são precisas e seriam melhor analisadas a partir de contextos individuais do ser humano, como suas condições físicas, biológicas, psicológicas e sociais, abordando um conjunto de informações subjetivas, sugerindo que indivíduos que apresentam a mesma idade cronológica podem apresentar diferentes estágios do envelhecimento. $\mathrm{O}$ tempo cronológico passaria a ser apenas uma contagem padronizada e, para compreender o ser em sua totalidade, estes apontamentos devem ser analisados na fundamentação de um conceito muito maior, definindo assim, a idade funcional (PAPALIA et al., 2006).

Weineck (2005) aponta que o envelhecimento é a soma de todas alterações ocorridas no espaço cro- 
nológico, sendo este então um fator que agrega essas transformações e nos permite, de forma individual e temporal, calcular o estágio do envelhecimento de cada um.

Por sua vez, a idade biológica proporciona uma melhor observação dos aspectos do envelhecimento. Essa mensuração depende do declínio e colapso graduais dos sistemas fisiológicos e cognitivos de cada ser. Conforme Carvalho Filho (2005, p.61), "todo ser vivo apresenta um padrão genético que condiciona seu desenvolvimento e envelhecimento." Com a chegada da velhice, as mudanças anatômicas ou morfológicas são principalmente as mais visíveis e manifestam-se em primeiro lugar (PAPALÉO NETTO, 2005).

A definição de idade psicológica pode ser identificada em duas interpretações. A primeira é propriamente similar à definição de idade biológica e se aplica na relação existente entre a idade cronológica e as habilidades tais como: percepção, raciocínio e memória, as quais podem demarcar o futuro potencial de comportamento cognitivo do indivíduo (NERI, 2005). Sabe-se que, mesmo durante o processo de envelhecimento normal, algumas capacidades cognitivas como a rapidez do processamento de informações e a memória diminuem naturalmente com a idade. Entretanto, a senilidade não é um componente obrigatório do envelhecimento. A segunda aplicabilidade da definição de idade psicológica tem relação com a percepção subjetiva de idade. Esta varia conforme cada indivíduo avalia a presença ou não de fenômenos biológicos, sociais e psicológicos do envelhecimento em comparação com outras pessoas de sua idade. Aumenta-se a tendência da realização de um retrospecto daquilo que se viveu e passa-se a pensar no tempo que ainda resta de vida (ARGIMON; STEIN, 2005).

Conforme Neri (2005), o envelhecimento social é caracterizado pelo processo de mudança de papeis e comportamentos, típico do momento da vida adulta em que o indivíduo tenta se adequar ao comportamento que a sociedade espera que ele tenha em determinada faixa etária. Witczak (2005) aponta que o afastamento do trabalho e a aceitação da aposentadoria geram sentimentos antagônicos, pois o indivíduo, ao mesmo tempo que experimenta o sentimento de liberdade, da livre utilização de seu tempo, encontra como obstáculo o medo da perda dos hábitos que o acompanharam por significativo período da vida.

De acordo com Papaléo Netto (2005), as modificações fisiológicas da senescência compreendem fatores de alteração intrínsecos, ou seja, aqueles que ocorrem no organismo do indivíduo independentemente de influência externa e fatores extrínsecos ou ambientais, compostos pelas alterações morfológicas decorrentes da dieta, do meio em que se vive e do estilo de vida, que tornam o organismo mais suscetível às intempéries da vida e que podem levá-lo à morte.

A dieta, o estilo de vida, e o exercício são fatores extrínsecos que afetam o processo de senescência. $O$ estilo de vida social e pessoal influencia de diversas maneiras no processo de envelhecimento. A análise do estado nutricional de idosos, avaliados pelo índice de massa corporal (IMC), apresenta estado de desnutrição em homens com idade mais alta, e maior propensão à obesidade em mulheres no início da velhice. (SPIRDUSO, 2005).

Ainda de acordo com Spirduso (2005), a capacidade funcional e manutenção da autonomia está relacionada a forma com que os idosos levaram a vida. Padrões de exercícios e de atividade física contribuem para as diferenciações individuais em faixas etárias específicas. A diminuição ou perda da autonomia estaria associada à redução da eficiência de alguns órgãos e sistemas que acabariam por afetar a capacidade funcional (AMORIM et. al 2002).

Entretanto, nem sempre é simples estabelecer os limites entre a senescência e a senilidade, ou seja, entre àquelas modificações que são peculiares ao processo do envelhecimento ou se são alterações advindas das morbidades mais comuns em idosos. Essa dificuldade se deve à grande variabilidade comportamental perante à idade e, também, ao fato de que os idosos não se apresentam com manifestações clássicas de alterações do organismo, como as que são apresentadas nas crianças, por exemplo (PAPALÉO NETTO, 2005).

Aspecto relevante que deve ser considerado e apresenta opiniões contraditórias é a ocorrência de demências. Com o passar dos anos, ocorre a perda de $0,2 \%$ de massa encefálica por ano de vida (ESIRI, 
2007). Essa perda poderia representar insuficiência das funções cerebrais que, em estágios avançados, levaria a um quadro demencial. Este argumento relaciona diretamente a alteração funcional à anatômica, entretanto, sem estudos comprobatórios. Em contraponto, estudos populacionais explanam que há preservação do intelecto e das funções mentais na senescência. Spirduso (2005) conclui então que as insuficiências das funções cerebrais não podem ser consideradas como consequência comum do processo de envelhecimento e, portanto, representaria o impacto de diversas morbidades que teriam sua ocorrência aumentada em idades mais avançadas.

\section{O ENVELHECER E A ATIVIDADE FÍSICA}

Estudos realizados por Hurley e Roth (2000) e Graves e Franklin (2001) evidenciam que as populações fisicamente ativas têm menor incidência de uma gama de doenças crônicas, dentre elas hipertensão arterial, obesidade, osteoporose, ansiedade e depressão, reconhecendo a atividade física como um importante fator promotor de saúde em todas as idades. Quando as pessoas já apresentam essas patologias, como é o caso de muitos idosos, a atividade física pode ser importante recurso para o tratamento, reduzindo a necessidade ou as doses de medicamentos em geral.

Gorzoni e Jacob Filho (2008) refletem que, embora as atividades aeróbias sejam adequadas para promoção da saúde cardiovascular, talvez não sejam as mais eficientes ou seguras, tanto de um ponto de vista cardiocirculatório quanto de alterações da estrutura musculoesquelética. Estudos realizados recentemente já apontam importantes efeitos protetores do sistema cardiovascular advindos do treinamento de resistência (BRAITH; STEWART, 2006).

A saúde musculoesquelética pode ser definida pela ausência de dores e uma boa funcionalidade sobre o olhar da biomecânica corporal. Mesmo afetado pela individualidade biológica, o envelhecimento sedentário tem como característica geral o agravamento de processos degenerativos no organismo humano, apresentando efeitos negativos nas cartilagens, tendões e músculos, além de intensificar a perda da massa óssea. A atividade física em geral apre- senta reflexos atenuantes, embora os exercícios mais eficientes nesses aspectos sejam os de resistência, porque estes levam em consideração diversos aspectos neurológicos, musculares e esqueléticos, fundamentais na manutenção da autonomia e bem-estar dos idosos (GRAVES; FRANKLIN, 2001).

A perda da capacidade funcional leva à insuficiência na realização das Atividades da Vida Diária (AVD'S) e das Atividades Instrumentais da Vida Diária (AIVD'S) onde, conforme Okuma (2012, p. 55):

\begin{abstract}
As primeiras referem-se às atividades de cuidados pessoais básicos, como vestir-se, banhar-se, levantar-se da cama e sentar-se numa cadeira, utilizar o banheiro, comer e caminhar uma pequena distância. As segundas referem-se às tarefas mais complexas do cotidiano e incluem, necessariamente, aspectos de uma vida independente, como fazer compras, cozinhar, limpar a casa, lavar roupa, utilizar meios de transporte e usar o telefone.
\end{abstract}

Portanto, as perdas no domínio cognitivo e das capacidades físicas contribuem para a redução da independência do idoso, limitando as possibilidades de vida confortável e satisfatória, aumentando o declínio de sua atuação na sociedade. Por conseguinte, essa limitação apresenta reflexos psicológicos (OKUMA, 2012). Segundo Schwanke et al. (2012, p.201), "a atividade física é um importante aliado na manutenção da independência, auxiliando a diminuir as perdas associadas ao envelhecimento e as consequências dessas perdas".

As atividades físicas de natureza predominantemente aeróbia têm sido o foco de atenção das pesquisas que relacionam aptidão física e saúde no idoso, devido sua associação na melhora de condições da saúde biológica e de certas doenças crônicas relacionadas com o sistema cardiorrespiratório. Entretanto, na prática, essa melhora seria satisfatoriamente explicada pela redução dos fatores de risco cardiovasculares do que com a capacidade do indivíduo de se manter funcionalmente independente. Ou seja, para o idoso realizar suas tarefas cotidianas como subir escadas, banhar-se ou carregar suas compras, ele não necessita apenas de aptidão cardiovascular, mas sim de um conjunto de capacidades como força muscular, resistência muscular localizada, flexibilidade, potên- 
cia, resistência e coordenação, conjunto denominado "aptidão corporal" pelo American College of Sports Medicine (ACSM) (OKUMA, 2012).

Dessa maneira, para se atingir objetivos específicos, determinados estímulos de atividade física serão necessários, tornando-a mais eficiente. $\mathrm{O}$ aprimoramento das qualidades de aptidão corporal tem sumária importância quando o objetivo é o de realizar grandes esforços, como no caso da prática esportiva. No entanto, os esforços para a prática das atividades básicas ou instrumentais da vida diária (AVD'S e AlVD'S) também exigem aptidão em níveis adequados para que sua realização seja possível e não represente fator de desconforto ou risco de lesões musculoesqueléticas e acidentes cardiovasculares (GORZONI; JACOB FILHO, 2008).

O equilíbrio corporal, a manutenção da postura e a marcha são afetados pela redução da força muscular. Para que a marcha seja possível, confortável e segura, a força é a aptidão mais importante. A resistência para caminhar depende da capacidade aeróbia, medida pelo limiar anaeróbio e estimulada pelo aumento da força muscular. Indivíduos treinados caminham com pouca ativação de unidades motoras, enquanto pessoas sedentárias utilizam muitas fibras para a marcha. Essa demanda de contração das fibras musculares implica na oclusão parcial de vasos sanguíneos intramusculares e assim algumas fibras não recebem sangue e oxigênio, gerando desconforto por acidose metabólica e fadiga precoce (GORZONI; JACOB FILHO, 2008).

A segurança das atividades físicas depende da adequação das sobrecargas às condições físicas individuais dos praticantes. Portanto, respeitando princípios de aplicabilidade e individualidade, a utilização de um simples programa de condicionamento físico, que estimule as aptidões importantes para uma vida diária confortável e segura, possibilitando a realização das AVD's e AIVD's, é vista como a escoIha mais adequada para o público idoso. Esses programas, por sua vez, apresentam diversas qualidades que os acompanham, citando dentre elas, a socialização, visto que muitas vezes as sessões de treinamento são realizadas em pequenos grupos, possibilitando ao idoso que ele se sinta companheiro de outro praticante, com objetivos em comum. Sendo assim, ocorre a melhora das interações sociais e interculturais, além da formação de novas amizades e ampliação das redes sociais, onde o contato entre diferentes gerações, por exemplo, diminuem o estereótipo das percepções sobre o envelhecimento (GORZONI; JACOB FILHO, 2008).

\section{PSICOMOTRICIDADE E gerontomotricidade}

Costa (2002) define que a psicomotricidade é uma ciência abstrata que estuda a humanidade através de suas relações corporais em movimento e em relação ao seu mundo interno e externo. Aborda também o processo de maturação psicológica, onde o corpo é a origem das aquisições cognitivas, afetivas e orgânicas. É sustentada basicamente por três pilares de conhecimentos: o movimento, o intelecto e o afeto, que, ainda de acordo com o autor citado (COSTA, 2002, p.17), fazem com que:

\begin{abstract}
A psicomotricidade baseie-se em uma concepção unificada da pessoa, que inclui as interações cognitivas, sensório-motoras e psíquicas na compreensão das capacidades de ser e de expressar-se, a partir do movimento, em um contexto psicossocial. Ela se constitui por um conjunto de conhecimentos psicológicos, fisiológicos, antropológicos e relacionais que permitem, utilizando o corpo como mediador, abordar o ato motor humano com o intento de favorecer a integração deste sujeito consigo e com o mundo dos objetos e outros sujeitos.
\end{abstract}

Nas palavras de Fonseca (2004, p. 10), as características da organização motora não podem ser separadas da organização psíquica, e esta por sua vez não é separável do mundo exterior, da realidade, da situação ou do conjunto de estímulos presentes porque, em psicomotricidade, o psíquico e o motor não são uma consequência linear um do outro, mas sim componentes complementares que interagem entre si, modulando a totalidade sistêmica, encarando corpo e motricidade como elementos essenciais da estrutura psicológica do Eu, onde, porventura,

[...] quando tais componentes não se encontram sistemicamente integrados, surge a disfunção psicomotora, uma síndrome e não uma doença, cuja origem não é especificamente orgânica, traumática 
ou hereditária. Trata-se do efeito ou reflexo corporal, postural, práxico ou condutivo de uma perturbação ou dificuldade psicológica que tende a manifestar-se em termos de desenvolvimento e de aprendizagem ou de adaptação psicossocial. (FONSECA, 2004 p. 11).

As práticas de intervenção psicomotora partem normalmente de uma prévia observação, com base na aplicação de uma bateria psicomotora, onde se constitui um perfil psicomotor intra-individual, espécie de ficha de inventário onde se apura as possibilidades ou dificuldades psicomotoras baseando-se nos fatores de tonicidade, equilíbrio, lateralização, noção de corpo, estruturação espaço-temporal, práxia global e práxia fina (micromotricidade), que no seu conjunto de fatores e subfatores constituem o sistema psicomotor humano (FONSECA, 2004).

Os estudos realizados com idosos por Levy (2000), apontam que o repertório de atividades psicomotoras estimula acima de tudo a consciência de um corpo que é expressão de um ser que pensa, sente, e que age em relação com os outros, com os objetos e consigo mesmo. Dessa maneira, a exploração do corpo é proposta com o objetivo de uma tomada de consciência para que haja melhor uso e consequentemente maiores possibilidades de expressão. Finalmente, um maior domínio corporal, ou seja, o desenvolvimento da autoconfiança reflete-se diretamente em mudanças na autoimagem, no aumento da autoestima, na funcionalidade e na manutenção da autonomia.

Dessa maneira, a psicomotricidade e a gerontologia de intervenção estão conceituadas muito próximas de si e vão ao encontro dos anseios da necessidade de movimento, característica presente no ser humano, compondo então a gerontomotricidade, que tem como objetivo a recuperação e a conservação de forma funcional das condutas psicomotoras, aprimorando o conhecimento universal de si e a eficácia das ações, principalmente das atividades da vida diária. Tem por meta o desenvolvimento integral do ser idoso, propondo, de forma equilibrada e harmoniosa, a relação entre a motricidade e o psiquismo. Proporciona o equilíbrio e a noção do espaço que o corpo ocupa, em sua totalidade, auxiliando na manutenção da saúde. Podemos fundamentá-la através de práticas individuais ou em conjunto, de acordo com o processo de envelhecimento corporal ou psicológico e da situação própria de cada idoso submetido à esta prática terapêutica. Portanto, gerontomotricidade é a prática dirigida ao idoso através da psicomotricidade (VASCONCELOS, 2003).

Estudo realizado com idosos institucionalizados demonstrou que após a intervenção do programa gerontopsicomotor houve alterações positivas de maior significância nas atividades de banho, alimentação, vestuário da parte superior e higiene corporal, sendo que estas foram as atividades em que houve maior foco durante o programa. As de menor impacto positivo ou sem alteração significativa compreendem o controle de esfíncteres, uso do vaso sanitário e locomoção, ou seja, deslocamentos que exigiam a saída do leito, já que as atividades não puderam ser realizadas com deslocamento além do leito hospitalar devido ao estado de saúde dos idosos avaliados. Foram utilizadas principalmente atividades de autocuidado, simulação e pouca mobilidade, proporcionando ao paciente que ele se sinta capacitado para outras realizações. Todavia, se comprovou melhora funcional através da estimulação psicomotora nesses idosos (OVANDO; COUTO, 2010).

A aplicação de atividades psicomotoras às pessoas idosas, através de uma reeducação psicomotora, exige cuidados especiais. É preciso pensar nas alterações anatômicas e fisiológicas que possam garantir uma margem de segurança, tanto aos idosos quanto ao profissional que aplicará o programa de atividades. Intervenções multidisciplinares são recomendadas, embora a orientação do programa seja de responsabilidade do educador físico. É aconselhável também a realização de cursos de especialização na área geriátrica, obtendo maiores conhecimentos específicos para o trabalho com o público idoso. Cabe também a este profissional exigir comprovações médicas, através de atestados, indicando as condições e limitações do idoso visando o planejamento de uma aula segura (BARRETO; SILVA, 2004).

É de suma importância planejar as atividades que serão realizadas. Lorda (2004), sugere alguns critérios básicos na programação de propostas em grupos ou individuais. Dentre elas, destacamos o respeito às diferenças dos componentes do grupo; o levar em con- 
sideração a falta de tradição desportiva e a progressiva perda de identificação com a imagem corporal real; ter objetivos do plano de gerontomotricidade correspondentes às necessidades e motivações das pessoas visadas, onde se permite que o participante explore todo o leque de possibilidades e a seguir escolha os meios e os modos de desenvolver as tarefas que se propõe; proporcionar momentos de motivação individual e ao grupo, onde as propostas de atividades devem ser coerentes ao plano geral, a fim de se manter continuidade e progressão e por fim, realizar avaliações prévias e periódicas durante a sequência do programa de atividades. $O$ professor de educação física deve ser claro nas explicações das atividades, se valendo de exemplos práticos, buscando sempre manter todos alunos em atividade, propondo metas que possam ser atingidas pelos participantes e estabelecendo regras que sejam cumpridas.

Durante a realização das atividades, o profissional deverá monitorar as situações que se desenrolam no ambiente, bem como as ocorrências fisiológicas individuais; fazer uso de diferentes posicionamentos, evitando ocorrência de sobrecarga em determinada região corporal; evitar movimentos bruscos, hiperflexões ou hiperextensões, além de rotações desnecessárias da coluna vertebral, posições de cabeça para baixo ou manobra de valsalva (fechamento da glote); utilizar materiais leves; não propiciar competições ou busca de performance excessivas; corrigir sempre a postura e permitir a interrupção das atividades por parte do aluno. Sob o aspecto de intervenção psicológica, o professor deve elogiar sempre, entretanto corrigindo e auxiliando no momento devido, demonstrando entusiasmo e interesse, agindo com honestidade perante ao aluno, respondendo todas as perguntas e durante atividades grupais, evitar ao máximo a ocorrência de constrangimentos diante dos companheiros (BARRETO; SILVA, 2004).

Dentre as atividades recomendadas aos idosos participantes do programa de gerontomotricidade estão o ato de rolar, deitado no solo ou sobre colchonetes, partindo de decúbito dorsal, com braços estendidos, cruzados no peito ou ao longo do corpo; engatinhar, de padrão cruzado ou com as pernas estendidas, estimulando o tônus postural; a marcha cruzada, consistida na movimentação alternada de pernas e braços, observando-se a postura corporal e o equilíbrio dinâmico do idoso; atividades de variação do equilíbrio, seja no solo ou em elevação, pois são exercícios que atuam sobre o córtex, o cérebro e o cerebelo, relacionando-se com as demais estruturas do sistema nervoso central. Ainda, deve se considerar o aspecto auditivo, sob a perspectiva de combinar atividades de caminhar e bater palmas em ritmo, ou agachar-se e levantar-se conforme o som de uma música, seja em jogos cooperativos ou rodas musicais; exercícios de aprimoramento manual, pois proporcionam a melhora da coordenação fina. Também é importante trabalhar com atividades de coordenação visual e motora, visto que podem ser trabaIhados aspectos de lateralidade, organização espaço-temporal e dinâmica corpórea geral, com exercícios que envolvam ações de pegar, tocar, arremessar objetos de pequeno porte, tocar e sentir as variações de tamanho, olhar para o companheiro e para o ambiente. Com essas atividades citadas por último, atua-se sobre o córtex visual e o auditivo, situados nos lobos occipital e temporal, respectivamente, além do córtex motor contro-lateral (BARROS, 2000).

A partir de uma elaboração com olhar atento aos princípios da gerontomotricidade, observa-se resultados positivos como os apresentados no estudo de Costa (2011), em que foi avaliado o nível de qualidade de vida em idosos submetidos a exercícios psicomotores como estratégia de educação em saúde. A amostra compunha idosos com idade média de 69,8 anos, $50,0 \%$ ativos e $50,0 \%$ de inativos, $90,0 \%$ mulheres e $10,0 \%$ homens. Os resultados demostraram que os idosos ativos apresentaram melhores valores em equilíbrio, elemento psicomotor avaliado, em comparação com os idosos inativos, apresentando diferenças significativas. Se valendo do teste de Berg, foi constatada uma diferença de 7,5 da pontuação da amostra entre os idosos ativos e inativos em relação ao equilíbrio e risco de quedas. Esses resultados comprovam que a pessoa idosa deve praticar exercícios físicos de forma regular, preferencialmente psicomotores, mantendo uma vida dinâmica com convívio social. A autora ainda conclui que " a abordagem da psicomotricidade para Terceira Idade parte do princípio do movimento corporal" (COSTA, 2011, p.112). 


\section{CONSIDERAÇÕES FINAIS}

Reunindo e analisando as informações sobre o envelhecimento sob a perspectiva da realização de atividades físicas e abordando os aspectos psicomotores dessas questões, pode-se estabelecer um rumo de observações que orientem a prática desses exercícios por parte dos idosos. São necessários o pensamento e a compreensão dos educadores físicos sobre as individualidades que se apresentarão durante a realização das atividades. As perdas oriundas do processo de envelhecimento são amplamente abordadas pela literatura. Costumam ser conceituadas através da variabilidade e complexidade das alterações orgânicas acarretadas como, por exemplo, a redução do equilíbrio estático e dinâmico e da mobilidade articular, modificações das funções fisiológicas e transformações psicológicas que sujeitam o indivíduo à ansiedade e à depressão.

Com o passar do tempo, o indivíduo em senescência apresenta modificações inevitáveis em seu organismo. Em vista disso e do aumento da longevidade humana, se tornam necessários novos estudos que contem com a participação de idosos, aliados fundamentais na melhor compreensão sobre os mecanismos fisiológicos do envelhecimento (CARVALHO FILHO, 2005).

$\mathrm{Na}$ tentativa de atenuar as ocorrências do enveIhecimento, a atividade física constitui um fator importante na manutenção da independência, diminuindo as perdas associadas a este processo, bem como suas consequências. As dificuldades de equilíbrio, as alterações na postura corporal e o controle dos movimentos prejudicam a execução das atividades de vida diária, aumentando o risco de quedas, que podem levar a fraturas e internações hospitalares. O mau condicionamento físico, a deficiência muscular, o tecido conjuntivo inflexível e o treinamento inadequado têm um impacto tanto na estática quanto no corpo em movimento (SCHWANKE et al., 2012).

Quando se trata de escolher as atividades adequadas ao público idoso, deve se levar em conta a preferência individual, sempre que possível. As atividades esportivas em geral podem ser muito úteis na promoção de qualidade de vida, mas a individualida- de deve ser respeitada. Muitas vezes, os idosos não possuem repertório motor necessário à sua prática ou sequer frequentaram ambientes esportivos durante a fase jovem de sua vida, apresentando dificuldades de socialização neste momento. Práticas esportivas também envolvem ocorrência de traumas musculoesqueléticos, além de um aumento desnecessário da frequência cardíaca. Portanto, respeitando os princípios de aplicabilidade e características individuais, a utilização de um simples programa psicomotor de condicionamento físico, que estimule as aptidões importantes para a realização das atividades da vida diária e das atividades instrumentais da vida diária, é vista como a escolha mais adequada para o público idoso onde, dessa maneira, abordamos a gerontomotricidade.

O profissional de Educação física responsável pela orientação das atividades deve estar ciente de que não está trabalhando apenas a saúde física de seus alunos, ele deve saber que cria um impacto social positivo na saúde mental deles e o quanto isto é fundamental na terceira idade. Sentir-se capaz de realizar as próprias tarefas e ser independente faz o idoso estar bem consigo mesmo e aceitar algumas limitações que possa apresentar, assim como seu novo papel na sociedade.

Certos cuidados devem ser tomados pelo educador físico durante o programa gerontopsicomotor, destacando o respeito às diferenças dos componentes do grupo, levando em consideração a falta de tradição desportiva e a progressiva perda de identificação com a imagem corporal real e, acima de tudo, ter objetivos do plano de gerontomotricidade correspondentes às necessidades e motivações das pessoas visadas. Através das análises dos estudos na construção deste artigo, já se verifica que a prática regular da gerontomotricidade trouxe bem-estar psicossomático nos indivíduos de terceira idade, tornando-a indicativa de uma maior percepção de qualidade de vida nos idosos ativos que nos inativos, proporcionando, principalmente, autonomia funcional nestes indivíduos. Entretanto, como os programas educacionais e de incentivo a prática de exercícios psicomotores para esta população ainda são pouco explorados quanto a promoção de saúde, cabe a nós educadores físicos e aos outros profissionais da área da 
saúde buscar efetivamente a iniciativa em projetos que mobilizem os idosos e os motivem a se manter em atividade.

Nesse sentido, entende-se que o objetivo principal da atividade física regular na terceira idade é o retardamento do processo inevitável do envelhecimento funcional, através da manutenção de um estado suficientemente saudável, para que o idoso possa dispor de autonomia e independência, sendo necessário ampliar e aprofundar as pesquisas com os idosos, propiciando um novo leque de possibilidades, de atividades e de vivências psicomotoras, onde não se espera simplesmente longevidade, mas também qualidade de vida.

\section{REFERÊNCIAS}

AMORIM P. R. S., M. MIRANDA et al. Estilo de vida ativo ou sedentário: impacto sobre a capacidade funcional. Rev. Bras. Cienc. Esporte, Campinas, v. 23, n. 3, p. 49-63; 2002.

ANDRADE, Maria Margarida de. Redação científica: elaboração do TCC passo a passo. São Paulo, SP: Factash, 2007.
ARGIMON, I. L., \& STEIN, L. M. Habilidades cognitivas em indivíduos muito idosos: um estudo longitudinal. Cadernos de Saúde Pública, 21 (1), 64-72; 2005.

BARRETO, Sidirley de Jesus; SILVA, Carlos Alberto da. Gerontomotricidade: condicionamento físico, consciência corporal e lazer na longevidade. Blumenau: Acadêmica, 2004.

BARROS, D. R. In MATTOS, Carlos A. F. Psicomotricidade: da educação infantil à gerontologia. São Paulo: Lovise, 2000. cap. 16 , p. $153-160$;

BENTO, J. O. Apontamentos da janela da minha idade. Porto: Universidade do Porto: Mimeo, 2002

BRAITH, R.W.; STEWART, K.J. Resistance exercise training: its role in the prevention of cardiovascular disease. Circulation, v.113, p.2642-2650, 2006;

CARVALHO FILHO, E. T. In PAPALÉO NETTO, Matheus. Gerontologia: a velhice e o envelhecimento em visão globalizada. São Paulo: Atheneu, 2005. cap. 5, p. 60-70;

COSTA, A. C. Psicopedagogia \& Psicomotricidade: Pontos de intersecção nas dificuldades de aprendizagem. 2.ed. Petrópolis: Vozes, 2002.
COSTA, Mayara Leal Almeida. Qualidade de vida na terceira idade: a psicomotricidade como estratégia de educação em saúde. Dissertação de Mestrado. Lisboa, 2011;

ESIRI, M. (2007), Ageing and the brain. J. Pathol., 211: 181-187.

FLECK, S. J.; KRAEMER, W. J. Fundamentos do treinamento de força muscular. 2 ed. Porto Alegre: Artmed; 2006;

FONSECA, V. Psicomotricidade, perspectivas multidisciplinares. Porto Alegre, Artmed, 2004;

GORZONI, M.L.; JACOB FILHO, W. Geriatria e gerontologia: o que todos devem saber. São Paulo: Roca, 2008;

GRAVES, J. E.; FRANKLIN, B. A. Resistance Training for Health and Rehabilitation. Champaign: Human Kinetics, 2001;

HURLEY, B.F.; ROTH S.M., Strength training in the elderly: effects on risk factors for age-related diseases. Sports Medicine, vol. 30, no. 4, pp. 249-268, 2000;

LEVY, D. In MATTOS, Carlos A. F. Psicomotricidade: da educação infantil à gerontogia. São Paulo: Lovise, 2000. cap. 17, p. 161-172;

LORDA, C. R. Recreação na Terceira Idade. Rio De Janeiro: Sprint, 2004.

MATTOS, Carlos A. F. Psicomotricidade: da educação infantil à gerontogia. São Paulo: Lovise, 2000.

NAHAS, M. V. Atividade física, saúde e qualidade de vida: Conceitos e sugestões para um estilo de vida ativo. Londrina, Editora Mediograf, 4a edição, 2006.

NERI, A. L. Qualidade madura no atendimento domiciliário. In Y. A. O. Duarte \& M. J. D. Diogo (Orgs.), Atendimento domiciliar: um enfoque gerontológico (pp.33-47). São Paulo: Atheneu; 2000.

NERI, A. L., CANÇADO, F. A. X., GORZONI, M.L. e ROCHA S.M. Tratado de Geriatria e Gerontologia. Rio de Janeiro: Guanabara Koogan, 340-343, 2002;

NERI, A. L. Palavras-chave em gerontologia. Campinas: Alínea, 2005;

OVANDO, Laura Maria Koopman; COUTO, Tatiana Vieira do. Atividades psicomotoras como intervenção no desempenho funcional de idosos hospitalizados. Mundo Saúde, v. 34 , n. 2, p. 176-182, 2010

OKUMA, Silene Sumire. 0 idoso e a atividade física: Fundamentos e pesquisa. Campinas - São Paulo. 6. ed. Papirus, 2012 
PAPALÉO NETTO, Matheus. Gerontologia: a velhice e o envelhecimento em visão globalizada. São Paulo: Atheneu, 2005:

PAPALIA, D. E., OLDS, S. W., \& FELDMAN, R. D. Desenvolvimento humano. Porto Alegre: Artmed, 2006.

SANTARÉM, J.M. In GORZONI, M.L.; JACOB FILHO, W. Geriatria e gerontologia: o que todos devem saber. São Paulo: Roca, 2008. cap. 7.2, p. 83-95; 2002.

SCHWANKE, C. H. A. et al. Atualizações em geriatria e gerontologia IV: aspectos demográficos, biopsicossociais e clínicos do envelhecimento. EDIPUCRS, Porto Alegre, 2012;

SPIRDUSO, W. W. Dimensões físicas do envelhecimento. Barueri, SP: Manole. 2005;

VASCONCELOS, Mylena Menezes. A psicomotricidade como promotora da qualidade de vida na terceira idade.
Revista Iberoamericana de Psicomotridad Y Técnicas Corporales. Número 12. Novembro de 2003.

VINCENT, K. R.; VINCENT, H. K. Resistance training for individuals with cardiovascular disease. J Cardiopulm Rehabil. 2006;26:207-216;

VINCENT, K.R., BRAITH R.W., FELDMAN R.A., KALLAS H.E., LOWENTHAL D.T. Improved cardiorespiratory endurance following 6 months of resistance exercise in elderly men and women. Arch Intern Med 2002; 162: 673-8;

WEINECK, Jürgen. Biologia do esporte. 7. ed. São Paulo: Manole, 2005;

WITCZAK, M. V. C. Envelhecer ao aposentar-se: discutindo a aposentadoria masculina, o envelhecer e o subjetivar. Dissertação de Mestrado não-publicada. Instituto de Psicologia, Universidade Federal do Rio Grande do Sul, Porto Alegre, RS, 2005. 